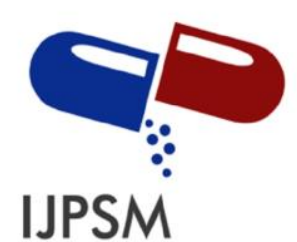

Shubhangi Parbhane et al, Int. Journal of Pharmaceutical Sciences and Medicine (IJPSM),

Vol.5 Issue. 12, December- 2020, pg. 11-22

ISSN: 2519-9889

Impact Factor: 3.426

\title{
SOLUBILITY ENHANCEMENT: MEANING AND TECHNIQUES
}

\author{
Shubhangi Parbhane; Aarti Belure; Aniket Garud; Mrs. Vibhavari Chatur* \\ Rasiklal M. Dhariwal Institute of Pharmaceutical Education and Research, Chinchwad, Pune-19 \\ E.mail Id: shubhangiparbhane22@gmail.com, aartibelure@gmail.com, \\ ani.pharmacology@gmail.com, vibhavaric@gmail.com \\ DOI: 10.47760/ijpsm.2020.v05i12.005
}

\begin{abstract}
The solubility enhancement process of hydrophobic drugs is crucial in formulation development to achieve the bioavailability and therapeutic action of the drug at the target site. About $40 \%$ of the new chemical entities that have been identified by pharmaceutical industry screening programs face numerous problems in the formulation and development stage due to the poor water solubility and low bioavailability. Drug solubility and bioavailability enhancement are the important challenges in the field of formulation of pharmaceuticals. The Bio pharmaceutics Classification System depicts that Class II and IV drugs have a low water solubility, poor dissolution, and low bioavailability. This review highlights on the importance, advantages, disadvantages, techniques of solubility enhancement.
\end{abstract}

Keywords: Solubility, BCS Classification, Solubility Enhancement Techniques, Solubility Enhancers.

\section{Introduction:}

Solubility, the phenomenon of dissolution of solute in solvent to give a homogenous system, is the main parameter to achieve desired concentration of drug in systemic circulation for desired pharmacological response. Low hydrophilicity is the major problem encountered with formulation development of new chemical entities and also for the generic development. The insufficient dissolution rate of the drug is the limiting factor in the oral bioavailability of poorly water soluble compounds. This review discusses BCS classification, carriers for solubility enhancement and different techniques for solubility enhancement. Different techniques are used to enhance the solubility of poorly soluble drugs which consist of micronization, nanonization, sonocrystallization, supercritical fluid method, spray freezing into liquid and lyophilisation, evaporative precipitation into aqueous solution, use of surfactant, use of co-solvent, hydrotropymethod, use of salt forms, solvent deposition, agents, modification of the crystal habit, co-crystallisation, complexation and drug dispersion in 


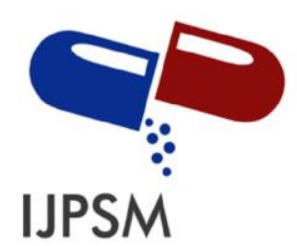

Shubhangi Parbhane et al, Int. Journal of Pharmaceutical Sciences and Medicine (IJPSM),

Vol.5 Issue. 12, December- 2020, pg. 11-22

ISSN: 2519-9889

Impact Factor: 3.426

carriers. ${ }^{(1,2)}$ Selection of solubility improving method depends on drug property, site of absorption, and required dosage form characteristic.

\section{Need of Solubility:}

On administration of an active agent orally, it must first dissolve in gastric and/or intestinal fluids before it gets permeated in the membranes of the GIT to reach systemic circulation. Drug absorption from the GI tract can be limited by a variety of factors, most significant contributor being poor aqueous solubility and poor membrane permeability of the drug molecule. Thus there are two areas of pharmaceutical research that focuses on improving the oral bioavailability of active agents which include, enhancing of solubility and dissolution rate of poorly water soluble drugs. The BCS is a scientific framework for classifying a drug substance depending on its aqueous solubility and intestinal permeability. ${ }^{(2,3)}$ As for BCS class II , IV drugs, rate limiting step is drug release from the dosage form and solubility in gastric fluid and not the absorption, thus increasing the solubility in turn increase the bioavailability for BCS class II , IV drugs.

\section{BCS Classification ${ }^{(1)}$}

Class I: High permeability and High solubility.

Formulation independent: The bioavailability of class I compounds is determined only by delivery of the drug solution to the intestine.

Examples: Loxoprofen, Benazepril, Sumatriptan, etc.

Class II: High permeability but low solubility

Formulation dependent: The bioavailability of class II compounds is limited by drug solubility/dissolution.

Examples: Aceclofenac, Valsartan, Nimesulide, Loratadine etc.

Class III: Low permeability but high solubility Dependent on barrier properties: The bioavailability of class III compounds is limited by intestinal permeability.

Examples: Atropine, Gabapentin, Topiramate, etc.

Class IV: Low permeability and low solubility

Formulation and barrier properties dependent: The bioavailability of class IV compounds is limited both by solubility/dissolution and intestinal permeability.

Examples: Hydrochlorothiazide, Meloxicam, Furosemide, etc.

\section{TECHNIQUES FOR SOLUBILITYE ENHANCEMENT OF POORLY SOLUBLE DRUG ${ }^{(9)}$}

\section{Chemical Modifications:}
1) Salt Formation
2) Co-crystallization
3) Co-solvency
4) Hydrotropy 


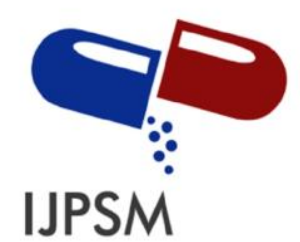

Shubhangi Parbhane et al, Int. Journal of Pharmaceutical Sciences and Medicine (IJPSM),

Vol.5 Issue. 12, December- 2020, pg. 11-22

ISSN: 2519-9889

Impact Factor: 3.426

5) Use of novel solubilizer

6) Nanotechnology

II. Physical Modifications:

Particle size reduction

a) Conventional method

b) Micronization

c) Nanosuspension

Modification of the crystal habit

Complexation

a) Polymorphs

a) Physical mixture

b) Kneading method

c) Co-precipitation method

Inclusion Complex Formulation Based Techniques

a) Kneading method

b) Freeze- drying Technique

c) Microwave irradiation method

Solubilisation by surfactants

a) Micro-emulsions

b) Self micro emulsifying drug delivery system

Drug dispersion in carriers

a) Solid solutions

b) Solid dispersions

i. Fusion Process

ii. Solvent Method

iii. Fusion solvent method

iv. Spray drying

v. Spray Freeze Drying Method

vi. Hot melt Extrusion vii. Dropping Method

\section{III. pH adjustment}

IV. Supercritical fluid process

V. Liquisolid technique

VI. Polymeric alteration 


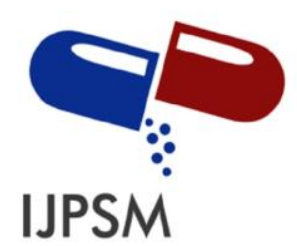

Shubhangi Parbhane et al, Int. Journal of Pharmaceutical Sciences and Medicine (IJPSM),

Vol.5 Issue. 12, December- 2020, pg. 11-22

ISSN: 2519-9889

Impact Factor: 3.426

\section{Size Reduction:}

There are Different techniques available to improve the solubility of poorly soluble drugs. Some of the approaches to improve the solubility are

\section{Micronization}

Particle size reduction leads to increase in the effective surface area resulting in enhancement of solubility and dissolution velocity of the drug.

Micronization technique is used to improve dissolution rates of drugs into the biological environment, in order to improve the oral bioavailability. Particle size reduction methods include recrystallization of the solute particles from solutions using liquid anti solvents, along with intensive techniques like crushing, milling, grinding, freeze drying and spray-drying. ${ }^{(1)}$

\section{Nanonization:}

Various nanonization strategies have emerged to increase the dissolution rates and bioavailability of numerous drugs that are poorly soluble in water. Nanonization broadly refers to the study and use of materials and structures at the nanoscale level of approximately $100 \mathrm{~nm}$ or less. Nanonization can result in improved drug solubility and pharmacokinetics, and it might also decrease systemic side-effects. For many new chemical entities with very low solubility, oral bioavailability enhancement by micronization is not sufficient because micronized product has the tendency to agglomerate, which leads to decreased effective surface area for dissolution, the next step is nanonization. ${ }^{(1)}$ There are many examples of nanonization of drugs.

\section{Nanocrystals:}

The term implies a crystalline state of the discrete particles, but depending on the production method they can also be partially or completely amorphous. Drug can be produced by bottom up technologies (precipitation methods) or alternatively by top down technologies (size reduction methods). At present, the most industrially feasible methods are the top down technologies, all products on the market are made by size reduction.

\section{Nanosuspension:}

Nanosuspensions are sub-micron colloidal dispersion of pure particles of drug, which are stabilised by surfactants. Nanosuspension technology has solved the problem of drugs which are poorly aqueous soluble and having less bioavailability. Stability and bioavailability of the drugs can be improved by the Nanosuspension technology. Nanosuspensions are prepared by using wet mill, high pressure homogenizer, emulsion, solvent evaporation, melt emulsification method and supercritical fluid techniques. Nanosuspensions can be delivered by oral, parenteral, pulmonary and ocular routes. 


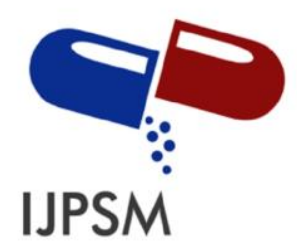

Shubhangi Parbhane et al, Int. Journal of Pharmaceutical Sciences and Medicine (IJPSM),

Vol.5 Issue. 12, December- 2020, pg. 11-22

ISSN: 2519-9889

Impact Factor: 3.426

\section{Nanoemulsions:}

Nanoemulsions are nonequilibrium, heterogeneous system consisting of two immiscible liquids in which one liquid is dispersed as droplets in another liquid. Emulsions with nanoscopic droplet sizes (typically in the range of 20-200 $\mathrm{nm}$ ) are often referred to as submicron emulsions. Nanoemulsions are composed of oil droplets dispersed in an aqueous medium and stabilized by surfactant molecules. The methods used for the production of nanoemulsions include $\mathrm{HPH}$, ultrasonication and spontaneous emulsification. Commercial products that are nanoemulsions include Estrasorb and Flexogan.

Supercritical Fluid (SCF): The numbers of applications and technologies involving supercritical fluids have also grown explosively. It has been known for more than a century that supercritical fluids (SCFs) can dissolve non-volatile solvents, with the critical point of carbon dioxide, which is most widely used supercritical fluid. It is safe, environmentally friendly, and economical. The low operating conditions (temperature and pressure) make SCFs attractive for pharmaceutical research. A SCF exists as a single phase above its critical temperature $(\mathrm{Tc})$ and pressure $(\mathrm{Pc}) .{ }^{(3)}$ SCFs have properties useful to product processing because they are intermediate between those of pure liquid and gas (i.e., liquidlike density, gas-like compressibility and viscosity and higher diffusivity than liquids). Moreover, the density, transport properties (such as viscosity and diffusivity), and other physical properties (such as dielectric constant and polarity) vary considerably with small changes in operating temperature, pressure, or both around the critical points . Hence, it is possible to fine-tune a unique combination of properties necessary for a desired application. These unique processing capabilities of SCFs, long recognized and applied in the food industry, have recently been adapted to pharmaceutical applications. Commonly used supercritical solvents include carbon dioxide, nitrous oxide, ethylene, propylene, propane, n-pentane, ethanol, ammonia, and water. ${ }^{(7)}$ Once drug particles are solubilised within SCF, they may be recrystallized at greatly reduced particle sizes. The flexibility and precision offered by SCF processes allows micronization of drug particles within narrow ranges of particle size, often to sub-micron levels. Current SCF processes have demonstrated the ability to create nanosuspensions of particles $5-2,000 \mathrm{~nm}$ in diameter. Several pharmaceutical companies, such as Nektar Therapeutics and Lavipharm, are specializing in particle engineering via SCF technologies for particle size reduction and solubility enhancement. ${ }^{(8)}$

\section{pH Adjustment:}

Adjustment of micro-environmental $\mathrm{pH}$ to modify the ionization behaviour is the simplest and most commonly used method to increase water solubility of ionisable compounds. As per $\mathrm{pH}$-partition hypothesis and equation, ionization of a compound is dependent on the $\mathrm{pH}$ of media and $\mathrm{pKa}$ of drug. ${ }^{(3,4)}$ The change in the ionic milieu can also result to in situ salt formation. However, this salt formation is infeasible for unionized 


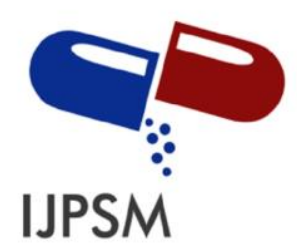

Shubhangi Parbhane et al, Int. Journal of Pharmaceutical Sciences and Medicine (IJPSM),

Vol.5 Issue. 12, December- 2020, pg. 11-22

ISSN: 2519-9889

Impact Factor: 3.426

compounds. ${ }^{(5,6)}$ The formed salts may also converse to respective acid or base forms in gastrointestinal-tract.

Solid Dispersions: These are generally prepared by solvent or co-precipitation method whereby both the guest solute and the solid carrier solvent are dissolved in a common volatile liquid solvent such as alcohols. The liquid solvent is removed by evaporation under reduced pressure or by freeze drying which results in amorphous precipitation of guest in a crystalline carrier. The basic difference between solid dispersion and solid solution is that the drug is precipitated out in an amorphous form in the former as opposed to crystalline form in the latter (Leuner and Dressman, 2000$)^{(12,15)}$

\section{Suitable Carriers for Solid Dispersion}

Many water soluble excipients are employed as carriers for solid dispersions. Following is the criteria that should be considered during selection of such carriers: higher water solubility, which improves wettability and enhance dissolution; high glass transition point leading to improved stability, minimal water uptake (reduces $\mathrm{Tg}$ ), soluble in common solvent with drug (solvent evaporation), relatively low melting point (melting process); capable of forming a solid solution with the drugsimilar solubility parameter. ${ }^{(12,15,16)}$

\section{Polyethylene glycol (PEG)}

PEG is generally water soluble polymer, which is formed by combining monomers of ethylene oxide having molecular weight usually between In the formulation of solid dispersions and solutions, PEGs with molecular weights ranging between 1500-20 000 are generally employed. As the molecular weight increases, they lead to increase in the viscosity. Drug to carrier ratio is one of the integral parts, which affects the properties of a solid dispersion.

\section{Polyvinylpyrrolidone (PVP)}

PVP results from polymerization of vinylpyrrolidone and have molecular weights ranging between 2500 and 3000 000. PVPs have a limited application for the preparation of solid dispersions by the hot melt method but due to their excellent solubility in an abundant variety of organic solvents, they are appropriate for the preparation of solid dispersions by the solvent method. Improved wettability and thereby an improved dissolution rate from a solid dispersion in PVP has been demonstrated. A profound disadvantage of PVPs is that they show poor aqueous solubility with increase in the chain length, additionally the high molecular weight of PVPs exhibit much higher viscosity at a given concentration. This was found in the case where the slower dissolution was observed from PVP K90 compared to PVP K12. It was attributed to the higher viscosity generated by PVP K90 in the diffusion boundary layer adjacent to the dissolving surface of the dispersion. ${ }^{(13)}$ Similar to PEG, solid dispersions prepared with high proportions of 


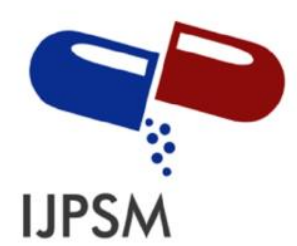

Shubhangi Parbhane et al, Int. Journal of Pharmaceutical Sciences and Medicine (IJPSM),

Vol.5 Issue. 12, December- 2020, pg. 11-22

ISSN: 2519-9889

Impact Factor: 3.426

PVP tends to high drug solubility and release rate than those with high proportions of drug.

\section{Carboxy Methyl Ethyl Cellulose (CMEC)}

CMEC belongs to the cellulose ethers, but it is resistant to dissolution under gastric or acidic conditions. It dissolves at $\mathrm{pH}$ above 5, with lowest dissolution $\mathrm{pH}$ being dependent on the grade of the CMEC. Amorphous solid dispersions of Nifedipine and Spironolactone showed massive enhancement in the dissolution rate of the drug at $\mathrm{pH}$ value of 6.8

\section{Polyvinyl Alcohol and polyvinyl acetate (PVA)/PVP copolymer}

These polymers belong to the polyvinyl group and are water soluble. The use of PVA/PVP copolymers as carriers in solid dispersions has been shown to lead an enormous increase in the drug release rate. When solid dispersions of Nifedipine were prepared with carrier mixtures consisting of Nicotinamide and PVP, hydroxyl propylmethylcellulose (HPMC) or PVA in a drug/Nicotinamide/polymer ratio of 1:3:1, those prepared with PVA dissolved 20 times faster than the drug alone. ${ }^{(14)}$ However, the other carriers, HPMC and PVP, yielded even better results .

\section{Crospovidone}

This polymer also belongs to a polyvinyl group which swells when dispersed in water. Although crospovidone does not dissolve in water, it can also be used as a carrier to improve drug release rate in solid dispersions. For example, solid dispersion of Furosemide with crospovidone led to an increase in the dissolution rate by a factor of 5.8 in comparison to either the drug powder or physical mixture of crospovidone. The mechanism of increase in the release rate was due to the presence of the drug in the amorphous form in the developed dispersion, which was proved by X-ray diffraction studies.

\section{Cellulose derivatives, HPMC}

Celluloses are naturally occurring polysaccharides, which can be further derivatized to form different carriers. The molecular weight of HPMC lies between 10,000 to 15,00,000 and are completely soluble in water. They are mixed ethers of cellulose where $16-30 \%$ of hydroxyl groups are methylated and about 4-32\% are derivatized with hydroxypropyl groups HPMC when used for the preparation of solid dispersion of Albendazole enhanced the release rate and bioavailability. It was further determined that HPMC was able to hinder the crystallization of Albendazole, and further improvement in release characteristics could be achieved by using HPMC and HPMCP in combination. Other drugs which exhibit faster release from solid dispersion in HPMC include poorly soluble weak acids Nilvadipine and Benidipine 


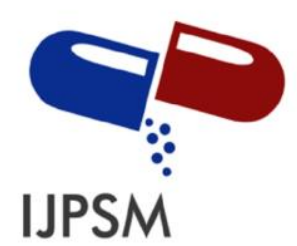

Shubhangi Parbhane et al, Int. Journal of Pharmaceutical Sciences and Medicine (IJPSM),

Vol.5 Issue. 12, December- 2020, pg. 11-22

ISSN: 2519-9889

Impact Factor: 3.426

\section{Polyacrylates and polymethacrylates}

Both polyacrylates and polymethacrylates are glassy substances which are formed by polymerization of acrylic and methacrylic acid. In pharmaceuticals, they are commonly utilized as coatings to modify the release of drug from the dosage form. Commonly the trade name Eudragit refers to them. Among all Eudragits, Eudragit E is frequently used to improve the release rate as it is soluble in all buffer at $\mathrm{pH}$ up to 5 and swells when $\mathrm{pH}$ is increased, while Eudragit L can be used when it is desirable to evade release in the stomach. Eudragit L has been effectively used to increase the dissolution of griseofulvin and spironolactone at a $\mathrm{pH}$ value of 6.8

\section{Urea}

Urea is the end product of human protein metabolism; it has a light diuretic effect and is regarded as nontoxic. Its solubility in water is good and it also exhibits fine solubility in several common organic solvents. In a bioavailability study of solid dispersions, it was shown that sulphathiazole was better absorbed in rabbits when given as a eutectic with urea. In case of ursodeoxycholic acid, it was found that the release rate from urea solid dispersions prepared by the hot melt method was more rapid as compared to other carriers, which were studied, like PEG 6000, Sugar, polyols and their polymers Even though sugars and associated compounds are highly water soluble and have less toxicity issues, yet they are less suitable than other carriers for the preparation of solid dispersions. The sugars have a high melting point, therefore restricting the applicability of hot melt method for preparation of solid dispersion. Similarly, due to its poor solubility in most organic solvents, it is difficult to form co-evaporates. In spite of these drawbacks, various attempts have been made to prepare solid dispersions with sugars and their derivatives. Mannitol is one such example, it has a melting point of $165-168^{\circ}$ and decomposes only above $250^{\circ}$ and has been employed in some cases to prepare dispersions by hot melt method

\section{Applications of solid dispersions}

1. To enhance the solubility of poorly soluble drugs thereby increase the dissolution rate, absorption and bioavailability.

2. To stabilize unstable drugs against hydrolysis, oxidation, recrimination, isomerisation, photo-oxidation and other decomposition procedures.

3. To reduce a side effect of certain drugs.

4. To hide unpleasant taste and smell of drugs.

5. Improvement of drug release from ointment creams and gels.

6. To avoid undesirable incompatibility. ${ }^{(12)}$

\section{Hydrotropy:}

Hydrotropyis solubilisation phenomenon whereby addition of large amount of a second solute results an increase in the aqueous solubility of another solute. Concentrated 


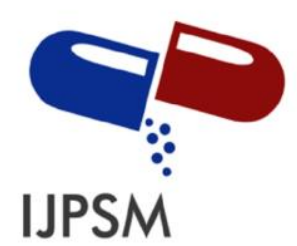

Shubhangi Parbhane et al, Int. Journal of Pharmaceutical Sciences and Medicine (IJPSM),

Vol.5 Issue. 12, December- 2020, pg. 11-22

ISSN: 2519-9889

Impact Factor: 3.426

aqueous hydrotropic solutions of sodium benzoate, sodium salicylate, urea, nicotinamide, sodium citrate and sodium acetate have been observed to enhance the aqueous solubility of many poorly water-soluble $\operatorname{drug}^{(17,18,19,20)}$

\section{Salt formation:}

Salt formation of poorly soluble drug candidates (weak acids and bases) has been a strategy for several decades to enhance solubility. It is an effective method in parenteral and other liquid formulations, as well as in solid dosage forms. Of approximately 300 new chemical entities approved by the FDA during the 12 years from 1995 to 2006 for marketing, 120 were in salt forms. In addition, out of the 101 approved salts of basic drugs, 54 salts were prepared with hydrochloric acid, indicating the hydrochloride was the predominant salt form. The aqueous solubility of an acidic or basic drug as a function of $\mathrm{pH}$ dictates whether the compound will form suitable salts. The $\mathrm{pH}$-solubility interrelationships also dictate what counter ions would be necessary to form salts, how easily the salts may dissociate into their free acid or base forms, what their dissolution behaviour would be under different GI $\mathrm{pH}$ conditions, and whether solubility and dissolution rate of salts would be influenced by common ion.

Several reviews have outlined general strategies and considerations for salt selection. The salt formation drug should have ionisable groups that will assist salt formation. The criteria used to select counter ion is as follows:

- There should be a minimum difference of 2-3 pKa units between the drug and the counter ion.

- Counter ions should decrease crystal lattice forces.

- It should be FDA approved or should have enough toxicological data to support the selection of the counter ion.

This technique has tremendous capability to enhance dissolution rate but it is grasped with disadvantages like approval of salts is a tedious task and also not useful for neutral molecules. ${ }^{(10)}$

\section{Cosolvents:}

Cosolvent system is a mixture of miscible solvents often used to solubilize lipophilic drugs. Currently, the water-soluble organic solvents are polyethylene glycol 400 (PEG 400), ethanol, propylene glycol, and glycerin. For example, Procardia (nifedipine) was developed by Pfizer contains glycerin, peppermint oil, PEG 400 and sodium saccharin in soft gelatin capsules. The water insoluble solvents include long-chain triglycerides (i.e. peanut oil, corn oil, soybean oil, sesame oil, olive oil, peppermint oil, hydrogenated vegetable oil and hydrogenated soybean oil), medium-chain triglycerides (Miglyol 812), beeswax, $d-\alpha$ - tocopherol (vitamin E) and oleic acid. ${ }^{(10)}$ 


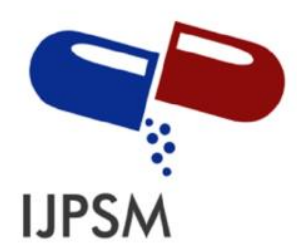

Shubhangi Parbhane et al, Int. Journal of Pharmaceutical Sciences and Medicine (IJPSM),

Vol.5 Issue. 12, December- 2020, pg. 11-22

ISSN: 2519-9889

Impact Factor: 3.426

\section{Cocrystals:}

Design of Molecular Crystals for Solubility Enhancement The design of molecular crystals that can be used as crystalline pharmaceutical materials is not infrequently aimed at enhancing the solubility and solution rate of drugs. To this end, a necessity arises to modify the surface and molecular packing of crystals contacting the medium.

Enhanced solubility is characteristic of unstable/ metastable polymorphic forms with retained molecular structure. Such polymorphs have a lower energy of crystal lattice, due to which they have a higher thermodynamic solubility. ${ }^{(21)}$ Number of studies have been published to show that polymorphous modification affects the solution rate and/or bioavailability of drug substance.

Example: Pharmaceutical co-crystals of carbamazepine (Tegretol)

Carbamazepine (CBZ) is an important antiepileptic drug that has been in use for over three decades. Oral administration of CBZ encounters multiple challenges, including low water solubility with high dosage required for therapeutic effect (i.e. $>100 \mathrm{mg} /$ day), dissolutionlimited bioavailability and auto induction for metabolism. In contrast to its simple molecular structure, CBZ exhibits complexity in its crystal forms. To date, four anhydrous polymorphs, a dihydrate, an acetone solvate, and two ammonium salts of CBZ have been identified. It is noted that, in the crystal structures of all these forms, the selfcomplementary nature of the amide group manifests itself in a predictable manner. Therefore, CBZ has been used as an ideal candidate to demonstrate how APIs can be converted to pharmaceutical co-crystals, and how these co-crystals could offer optimized physicochemical properties over existing forms of an API. Two strategies have been adopted for co-crystal formation of CBZ. One crystal engineering strategy is to employ the peripheral hydrogen bonding capabilities that are not engaged in the pure form of CBZ. A second strategy for co-crystallization of CBZ involves breakage of the CBZ amide-amide dimer and formation of a supramolecular heterosynthon between $\mathrm{CBZ}$ and a co-crystal former. Both strategies are successful and have afforded a number of CBZ co-crystals that exhibit improved physicochemical properties. For example, the CBZ saccharin co-crystal shows significantly improved physical stability (i.e. only one co-crystal form with equivalent chemical stability to the anhydrous polymorph has been identified after sophisticated form screening). In addition, the CBZ saccharin co-crystal possesses favourable dissolution properties, suspension stability, and pharmacokinetics using dog models. The pharmacokinetic study reveals that the CBZ saccharin co-crystal exhibits a higher $\mathrm{C}_{\max }$ and comparable $\mathrm{T}_{\max }$ when compared with the marketed form, Tegretol. In short, the CBZ saccharin co-crystal appears to be superior to existing crystal forms of CBZ in the following respects: stability relative to the anhydrous polymorph of CBZ, favourable dissolution and suspension stability, favourable oral absorption profile in dogs. ${ }^{(22)}$ 


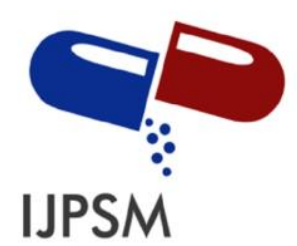

Shubhangi Parbhane et al, Int. Journal of Pharmaceutical Sciences and Medicine (IJPSM),

Vol.5 Issue. 12, December- 2020, pg. 11-22

ISSN: 2519-9889

Impact Factor: 3.426

Conclusion: With the help of this article we would like to conclude that the solubility of the drug is the most important factor that controls the formulation of the drug as well as therapeutic efficacy of the drug. The various techniques that are described above alone or in combination can be used to enhance or improve the solubility of drug. We can opt any of the drugs for its action by means of altering its solubility with respect to above techniques if that particular drug faces any kind of solubility issues.

\section{References}

1) Patel Chirag J., Asija rajesh., Asija Sangeeta., Different method of enhancement of solubilisation and bioavailability of poorly soluble drug: A recent review Pharma tutor 2012, Reference ID-pharma-tutor-art-1424.

2) Martin Alfred N., Patrick J. Sinko., Yashveer Singh., Martin's Physical Pharmacy and Pharmaceutical Science, $6^{\text {th }}$ Edition 2011: 180-196.

3) Sandeep Kumar., Pritam Singh ,Various Techniques For Solubility Enhancement :An Overview ,The Pharma Innovation Journal ,2016; 5(1) :23-28.

4) Lachman L., Liberman H.A., Kanig J.L., The theory and practice of industrial pharmacy, 1990, $3^{\text {rd }}$ Edition, 76 (1) : 171-196.

5) Allen L.V., Popovich N.G., Ansel H.C., Ansel's Pharmaceutical dosage forms and drug delivery systems, $8^{\text {th }}$ Edition, 2005:100- 101.

6) Venkatesh S.Y., Rao V., Anderson B. D., Intrinsic solubility estimation and pHsolubility behaviour of an extremely hydrophobic diprotic acid, Pharmaceutical Research, 1996; 13(10):1453-1459

7) G. Sunkara., U. B. Kompella., Drug delivery applications of supercritical fluid technology, Volume 2, 2002: 44-50.

8) L. Manna., M. Banchero., D. Sola., A. Ferri., S. Ronchetti., S. Sicardi., Impregnation of PVP micro particles with ketoprofen in the presence of supercritical CO2, Journal of Supercritical Fluids , 42, ISSN- 32007: 378-384.

9) Sneha Jagtap., Chandrakant Magdum., Dhanraj Jadge., Rajesh Jagtap., A review: Solubility Enhancement Technique, 10(9): 2206.

10) Yogesh S. Thorat., Indrajeet D. Gonjari., Avinash H. Hosmani., Solubility Enhancement Techniques A review on conventional and novel approaches, International Journal of pharmaceutical science and research, ISSN0-975-8232: 2501-2513.

11) Varun Raj Vemula., Venkateshwarlu Lagishetty., Srikanth lingala., Solubility Enhancement Technique, 5(1), 2010: 41-42

12) A. N. Patil., D. M. Shinkar., R. B. Saudagar., A review article: Solubility Enhancement By Solid Dispersion, Inteernational Journal of current Pharmaceutical Research , 9(3), 2017:15-18 


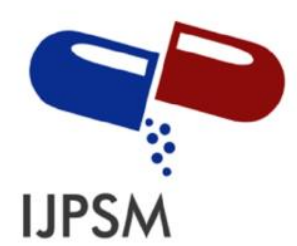

Shubhangi Parbhane et al, Int. Journal of Pharmaceutical Sciences and Medicine (IJPSM),

Vol.5 Issue. 12, December- 2020, pg. 11-22

ISSN: 2519-9889

Impact Factor: 3.426

13) Ludwigshafen: BASF, Buehler V., SolubleKollidon Grades (Povidone, Polyvidone): Tablet Coatings, Kollidon:Polyvinylpyrrolidone for the Pharmaceutical Industry, 1999: 106-15.

14) Wade A., Weller P J., Handbook of pharmaceutical excipient. American pharmaceutical association, $2^{\text {nd }}$ Edition, 1994 : 392-99.

15) Muhammad J. Habib., Pharmaceutical Solid Dispersion Technology, Technomic Publication, $2000: 65$

16) G. Singh., I. Kaur., G. D. Guptaand., S. Sharma., Enhancement of the Solubility of Poorly Water Soluble Drugs through Solid Dispersion: A Comprehensive Review ,79(5),2017: 674-685

17) Rajwardhan Reddy. M., Prasanna Kumar. D., Sreenivasulu. K., P.V.S. Srikanth., B. Brahmaiah., Sreekanth N., A Review On Hydrotropy , 2013; 2(4) : 5-6

18) A. A. Rasool., A. A. Hussain., L. W. Dittert., Solubility enhancement of some water-insoluble drugs in the presence of nicotinamide and related compounds, Journal of Pharmaceutical Sciences, 1991: 387-393.

19) A. A. Badwan., L. K. El Khordagui., A. M. Saleh., S. A. Khalil., The solubility of benzodiazepines in sodium salicylate solution and a proposed mechanism for hydrotropic solubilisation, International Journal of Pharmaceutics, 13,1983: 67-74.

20) B. K. Roy., S. P. Moulik., Functions of hydrotropes (sodium salicylate, proline, pyrogallol, resorcinol and urea) in solution with special reference to amphiphile behaviours, Colloids and Surfaces , 2002; 203 : 155-166.

21) Russian Journal of General Chemistry, 2014, 84(2), ISSN 1070-3632 : 407-414.

22) V Yadav., A.S.Shate., A.P. Dabke., P.V. Kulkarni., S.S. Sakhare., Co-Crystal: A Novel Approach to Modify Physicochemical Properties Of Active Pharmaceutical Ingredient, 71(4),2009 : 359-370. 